\title{
Protective Immunity in Bancroftian Filariasis \\ Selective Recognition of a 43-kD Larval Stage Antigen by Infection-free Individuals in an Endemic Area
}

\author{
D. O. Freedman, T. B. Nutman, and E. A. Ottesen \\ Laboratory of Parasitic Diseases, National Institute of Allergy and Infectious Diseases, Bethesda, Maryland 20892
}

\begin{abstract}
There is little information about naturally occurring protective immunity in individuals living in areas endemic for lymphatic filariasis, though an immunologically hyperresponsive, uninfected group of "endemic normal" individuals that may be immune has been previously recognized. To analyze the nature of the hyperresponsiveness and its potential relation to a state of protective immunity in such individuals, strict clinical, parasitological, and serological criteria were applied to select seven "infection-free"' endemic normal individuals (ENs) from a population of $\mathbf{4 5 9}$ persons resident in an area heavily endemic for bancroftian filariasis. Immunoblot analysis was used to compare the qualitative antigen recognition patterns of these endemic normal individuals to those of a group of 12 clearly infected microfilaremic individuals (MFs) from the same endemic area. Though immunoblot analysis using microfilarial and adult stage filarial antigens revealed no distinct differences in antigen recognition patterns between the two groups, when responses to infective larval stage antigens were assessed, 7/7 $(100 \%)$ of the ENs were found to recognize a $43-\mathrm{kD}$ antigen that was recognized by only $1 / 12(8 \%)$ of the MFs. These findings are consistent with the concept that recognition of unique larval antigens may induce protective immunity to human filarial parasites and they identify a candidate immunogen for further functional assessment.
\end{abstract}

\section{Introduction}

The nematodes Wuchereria bancrofti and Brugia malayi, causative agents of the lymphatic filariases, infect approximately 100 million people throughout the tropics (1). Infection of the human host occurs when third stage larvae (L3) ${ }^{1}$ of the parasite enter the body following the bite of an infected vector mosquito. The larvae mature over a period of months into lymphatic dwelling adult worms that release microfilariae into the host's bloodstream and initiate the adenolymphangitis and chronic lymphedema characteristic of these infections.

While there is no direct evidence demonstrating the existence of protective immunity in humans, the presence in all

Address reprint requests to Dr. Freedman, Laboratory of Parasitic Diseases, Building 4, Room 126, National Institutes of Health, Bethesda, MD 20892.

Received for publication 17 November 1987 and in revised form 12 July 1988

1. Abbreviations used in this paper: BmA, Brugia malayi adult antigen; DEC, diethylcarbamazine; EN, endemic normal individual; L3, infective larvae; $\mathrm{MF}$, asymptomatic microfilaremic individual; $\mathrm{mf}$, microfilariae; NCP, nitrocellulose paper; PC, phosphocholine; $\mathrm{Wb}, W$. bancrofti.

The Journal of Clinical Investigation, Inc.

Volume 83, January 1989, 14-22 endemic areas of a proportion of the adult population that is amicrofilaremic and has no clinical evidence of current or past lymphatic infection despite constant and lifelong exposure to infective L3's (2) suggests that protective immunity to lymphatic filariasis may develop naturally in some individuals. There is additional indirect evidence provided by cross-sectional studies that show microfilaria rates in endemic areas usually increasing until about age 30 (2), but thereafter remaining constant or even declining.

The most common strategy for studying protective immunity not only to parasites but to other infectious agents as well has involved the use of animal models; indeed, the recent successes in both schistosomiasis (3) and falciparum malaria (4) attest to the value of such an approach. For W. bancrofti infection, however, no good laboratory animal models have yet been developed, though other similar lymphatic-dwelling filarial parasites (including $B$. malayi) can be maintained in a number of experimental hosts (5). In these, the induction of protective immunity to challenge infection can be demonstrated both in previously naive animals immunized once with attenuated larvae, and, in one model, in microfilaremic animals who have been repeatedly inoculated with intact Brugia larvae every $10 \mathrm{~d}$ for extended periods of time (6).

The study of protective immunity in human populations infected with lymphatic filariasis has been hampered by the following: the difficulty in distinguishing from among the apparently uninfected individuals those who are truly immune and those harboring occult infection; and an inability to evaluate resistance to reinfection among the large numbers of individuals who have clinical manifestations of filarial infection yet whose relatively high degree of immune responsiveness to parasite antigens might reflect a state of "concomitant immunity" to the parasite. We have attempted to circumvent these problems in the present study by adopting a strategy to document in a detailed, comparative manner potential immunologic determinants of protective immunity to $W$. bancrofti by examining two rigorously definable groups of individuals living in the same highly endemic area (Mauke, Cook Islands), one clearly nonimmune and the other at least not infected (by all current criteria) and thus "putatively immune." The first group, nonimmune, was comprised of individuals who were asymptomatic but had $W$. bancrofti microfilariae circulating in the blood; such microfilaremics (MF) generally manifest profound and specific humoral (7-9) and cellular hyporesponsiveness $(10,11)$ to parasite antigens when compared to all other groups resident in endemic areas. The second group, by contrast, was also asymptomatic, had no circulating microfilariae and was infection free by all the relevant clinical and laboratory criteria that could be applied with our present state of understanding; such individuals may well have protective immunity to the parasite and were here regarded as putatively immune endemic normals (ENs). Similar individuals have previously been shown to have significantly greater quantitative cellular and humoral immune responses to parasite anti- 
gens than those found in MFs (7) and might be expected to recognize antigens capable of inducing protective immune responses.

Sera from all study subjects were analyzed by immunoblotting with adult, microfilarial, and infective larval extracts to define antigen recognition patterns that showed qualitative differences between the putatively immune group and those with patent bancroftian filariasis.

\section{Methods}

Study population. 459 of 650 residents of Mauke, Cook Islands, an area hyperendemic for subperiodic bancroftian filariasis, were surveyed as previously described (12). A history, physical examination and two membrane blood filtrations (Nuclepore Corp., Pleasanton, CA) identified 68 individuals as candidates for the present study; the infection status of these 68 was studied intensively. None of the 68 had received diethylcarbamazine (DEC) in the previous $10 \mathrm{yr}$, though each of these individuals subsequently received DEC as per the protocol described (10) and all were monitored for characteristic posttreatment reactions.

Serum specimens. Serum from all study patients was frozen in liquid nitrogen within 2-3 h of collection and maintained in liquid nitrogen or at $-70^{\circ} \mathrm{C}$ until being analyzed. Normal human sera (NHS) were collected from North Americans never exposed to human filarial parasites. For use as a positive control a pool of sera with a high titer of antifilarial antibody (Wb serum pool) was formed from sera of 10 individuals with various clinical manifestations of $W$. bancrofti infection.

Antigen preparation. Because Wuchereria bancrofti cannot be maintained in laboratory animals, soluble antigenic extracts were prepared from three different life cycle stages of the closely related human filarial parasite Brugia malayi, maintained in jirds (Meriones unguiculatus) (13). Adult worms and microfilariae were obtained from the peritoneal cavity of the infected jirds while infective larvae (L3) were dissected from Aedes aegypti mosquitoes fed on the jirds. All parasites were washed extensively in $\mathrm{PBS}$ and frozen at $-70^{\circ} \mathrm{C}$ until ready for use (supplied by J. McCall, University of Georgia, Athens, GA).

To prepare antigen for immunoblot analysis parasites of each individual life cycle stage were thawed, lyophilized, and suspended in extraction buffer ( $50 \mathrm{mM}$ Hepes, $100 \mathrm{mM}$ glycine, $\mathrm{pH} 7.2$ ) with the following protease inhibitors: EDTA, $1 \mathrm{mM} ; \mathrm{n} \alpha$-p-tosyl-L-lysine chloromethyl ketone (TLCK), $0.2 \mathrm{mM}$; leupeptin, $0.05 \mathrm{mM}$; and p-nitrophenyl-p-guanidinobenzoate (NPGB), $0.025 \mathrm{mM}$ (all from Sigma Chemical Co., St. Louis, MO). Adult and L3 parasites were homogenized in a hand-held glass tissue homogenizer (Thomas Co., Philadelphia, PA), and the microfilariae were sonicated in extraction buffer. Cetyltrimethylammonium bromide (Sigma), at a final concentration of $1 \%$, was added, and the parasites were incubated overnight at $4{ }^{\circ} \mathrm{C}$ with constant agitation. After centrifugation at $100,000 \mathrm{~g}$ for $1 \mathrm{~h}$ the supernatants were dialyzed against PBS ( $\mathrm{pH} \mathrm{7.4)}$ ) and protein determinations were performed (14).

The soluble antigen $(\mathrm{BmA})$ derived from adult $B$. malayi used for filaria-specific antibody determinations and for the circulating antigen assays was prepared as a PBS extract of homogenized worms at $4^{\circ} \mathrm{C}$, as described previously (10).

Soluble mosquito antigen (kindly supplied by J. McCall) was derived from whole uninfected Aedes aegypt $i$ that had been homogenized and extracted into RPMI 1640. The supernatant from $10,000 \mathrm{~g}$ centrifugation of the preparation was concentrated (Centricon-10; Amicon Corp., Danvers, MA), and chromatographed against PBS on a G-25 Sephadex column (PD-10; Pharmacia Fine Chemicals, Piscataway, NJ).

Quantitative antibody assessment. Filaria-specific IgG was determined in an ELISA using BmA as previously described (15). The Wb serum pool was assigned arbitrary units of specific antibody and was used for generation of calibration curves against which all patient sera were compared for their content of antifilarial antibodies. Filaria spe- cific IgE levels were also determined as described (16) in a solid-phase RIA. Total serum IgG was assessed by radial immunodiffusion with plates and standards from Meloy Laboratories (Springfield, VA). RIST or PRIST kits (Pharmacia) were used for determining total serum IgE.

Circulating parasite antigen determination. Phosphocholine (PC)containing circulating parasite antigen was determined using a noncompetitive ELISA, as previously described (17). Briefly, affinity-purified polyclonal anti-BmA coated onto microtiter plate wells was used as the trap for circulating antigen present in serial twofold dilutions of patient sera beginning at 1:1. After appropriate incubation and washing, the monoclonal antibody CA10i (with PC specificity) was added. After further incubation, the OD of each sample was determined after the addition of alkaline phosphatase conjugated goat anti-mouse Ig and phosphatase substrate. A standard curve, to which all test sera were compared, was generated for each plate by adding a known amount of BmA to NHS and diluting twofold across the plate. Every one of a group of 15 normal North American control sera run in parallel with the patient samples had undetectable levels $(<10 \mathrm{ng} / \mathrm{ml})$ of circulating parasite antigen.

Qualitative analysis of $\operatorname{IgG}$ antibody by immunobloting. SDSPAGE of all antigens was performed according to the method of Laemmli (18) in a 5-15\% gradient gel (or $10 \%$ straight gel for L3 experiments) with a $4 \%$ stacking gel. Acrylamide to bisacrylamide ratios were 37.5:1 throughout. Before electrophoresis all antigens were diluted $1: 1$ with sample buffer $(60 \mathrm{mM}$ Tris $\mathrm{HCl}, \mathrm{pH} 6.8$, containing 70 $\mathrm{mM}$ SDS and 5\% 2-mercaptoethanol) and boiled for $5 \mathrm{~min}$. Molecular weight standards (Bethesda Research Laboratories, Gaithersburg, MD) were run in each gel.

Antigens were transferred to nitrocellulose paper (NCP) as described by Towbin (19) using a Trans-blot cell (Bio-Rad Laboratories, Richmond, CA) at $150 \mathrm{~mA} / \mathrm{gel}$ for $16 \mathrm{~h}$ at $4^{\circ} \mathrm{C}$. For each experiment separate sheets of NCP for incubation with EN sera and separate sheets for incubation with MF sera were prepared simultaneously and in parallel. The NCP was blocked with $3 \%$ BSA in wash buffer $(50 \mathrm{mM}$

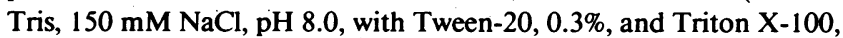
$0.05 \%$.) for $1 \mathrm{~h}$, washed, cut into strips, numbered, and placed in slotted plastic trays. The antigen strips were incubated individually with $5 \mathrm{ml}$ of a 1:50 dilution of patient sera at room temperature for $2 \mathrm{~h}$ and then washed six times in wash buffer. Either $5 \mathrm{ml}$ of ${ }^{125}$ I-labeled goat anti-human IgG (New England Nuclear, Boston, MA) at a concentration of $1.0 \times 10^{6} \mathrm{cpm}$ in $20 \mathrm{ml}$ wash buffer with $5 \%$ FCS (BmA and $\mathrm{L} 3$ experiments) or $5 \mathrm{ml}$ of alkaline phospatase conjugated goat anti-human IgG (Jackson Immunoresearch, Avondale, PA) at a concentration of $1: 2,000$ in wash buffer was added to each strip (microfilarial antigen). After overnight incubation at room temperature, strips were washed $(6 \times 15 \mathrm{~min})$. When the radioactive second antibody was used, the positive bands were visualized autoradiographically using Kodak XAR5 film in a Kodak X-Omat cassette (Eastman Kodak, Rochester, NY). When the alkaline phosphatase conjugate was used, the strips were developed using a BCIP/NBT substrate (Kirkegaard and Perry, Gaithersburg, MD). Relative molecular weights were assigned to each band based on its mobility in comparison to the known standards. The $\mathrm{Wb}$ serum pool and NHS were run with each immunoblot to act as positive and negative controls, respectively.

Statistics. Student's $t$ test using unpaired samples was used to compare the two patient groups in Table I. Geometric means were used for all calculations.

The significance of the difference in the rate of recognition of each individual antigen of a life cycle stage was tested by a Fisher's exact test and corrected by a Bonferroni inequality (20), which controls for the total number of comparisons made at that life cycle stage.

\section{Results}

\section{Definition of clinical groups}

To define a putatively immune, infection-free group of ENs for further immunologic evaluation, individuals who had been 
amicrofilaremic on the screening survey and who had no historical or physical indication of filarial infection after further detailed examination were subjected to seven separate Nuclepore filtrations at different times of day. None of the 12 individuals who met these initial criteria had any posttreatment reaction indicative of filarial infection when subsequently given DEC and monitored closely for the succeeding $4 \mathrm{wk}$. To select further for those among these 12 who were most likely to be infection free we utilized a recently developed assay to measure circulating filarial antigen in the sera of these individuals. Interestingly, only 7 of the 12 had completely negative $(<10 \mathrm{ng} / \mathrm{ml}$ ) circulating antigen assays (Fig. 1); these 7 were finally selected to constitute the EN group. The 12 individuals selected for inclusion in the MF group were microfilaremic on at least two separate occasions and were free from past or present lymphatic obstruction both on historical inquiry and on complete physical examination. In marked contrast to the EN group all persons in the MF group had positive circulating filarial antigen assays (Fig. 1) with a geometric mean antigen level of $1,458 \mathrm{ng} / \mathrm{ml}$ (range 100-8,000).

Table I shows the clinical data of the 7 ENs and 12 MFs selected for detailed immunologic assessment. There was no significant difference in the age distribution of the two groups, but there was a disproportionate number of females in the EN group (6/7), along with corresponding differences in occupations. When the entire population of the island was stratified by occupational exposure (12), however, there were no associated differences in prevalence of disease or infection. Though no entomologic studies were carried out, both the size of the island ( $2 \times 4$ miles maximum diameter) and the fact that all subjects had resided for their entire lives on the island ensured similar intense and prolonged exposures to the mosquito vector for all individuals.

All persons in the present study had one or more intestinal helminths on stool examination.

\section{Immunoglobulin levels}

There was no statistically significant difference in total serum IgG levels between the ENs and the MFs (Table I) but the geometric mean levels of filaria-specific IgG were significantly higher $(P<0.001)$ in the apparently uninfected ENs than in the infected MF group.

All individuals had high levels of total serum IgE (Table I), and there was no significant difference between the levels of

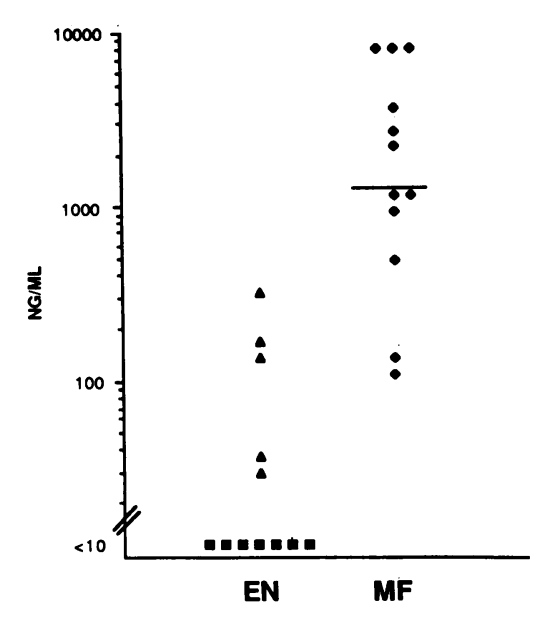

Figure 1. Circulating filarial antigen levels in the sera of the putatively infection-free normals (ENs, squares) and in the microfilaremics (MFs, closed diamonds) as detected by ELISA. The five individuals excluded from the EN group (see text) are represented by the triangles. Results are expressed as $\mathrm{ng} / \mathrm{ml}$ antigen equivalents of $\mathrm{BmA}$ (16); lower limit of assay's sensitivity 10 $\mathrm{ng} / \mathrm{ml}$. the two study groups. For filaria-specific IgE (Table I), however, again levels were significantly higher $(P<0.001)$ in the EN group than in the MF group.

\section{Antigen recognition by $\operatorname{Ig} G$ antibody in ENs and $M F S$}

To determine whether the apparently uninfected (putatively immune) ENs were producing protective IgG antibodies against individual filarial antigens not recognized by the infected MF patients, we performed immunoblot analyses using parasite antigens from three different life cycle stages. None of these antigens (adult, microfilarial, or infective larval stage) were recognized by the "negative control" serum, but a broad spectrum of antigens was recognized by the $\mathrm{Wb}$ positive control serum pool (Fig. 2).

Adult and microfilarial antigens. When patient sera were reacted with $B$. malayi adult antigen, individuals showed complex antigen recognition profiles. To define a potentially protective antigen recognized uniquely (or by close to $100 \%$ ) by the EN group and not at all (or by close to $0 \%$ ) by the MF group, we prepared a frequency-response analysis of the immunoblot findings (Fig. $3 A$ ). As seen, there was an overall similarity of adult antigen recognition patterns between the two groups. 21 of 27 antigens were recognized by roughly equivalent percentages of patients in each group. There was some difference in the frequency of antigen recognition between the ENs and the MFs when antigens of $15 \mathrm{kD}(100 \%$ ENs vs. $50 \% \mathrm{MFs}), 86 \mathrm{kD}(57 \%$ ENs vs. $17 \% \mathrm{MFs})$ and $88 \mathrm{kD}$ ( $71 \%$ ENs vs. $8 \%$ MFs) were compared but none of these differences reached statistical significance. Antigens at 24, 25, and $45 \mathrm{kD}$ were each recognized by only a few individuals in either one of the two groups; however, no antigen was recognized selectively by the ENs and not by the MFs.

Fig. $3 B$ shows the frequency-response analysis seen when reactivity of patient serum was similarly assessed with microfilarial antigen. Here, 25 of 36 antigens were recognized by approximately equivalent percentages of the patients. Antigens of 23 (100\% ENs vs. $58 \% \mathrm{MFs})$ and $85 \mathrm{kD}$ (100\% ENs vs. $50 \%$ MFs) showed small (not statistically significant) differences in the frequency of antigen recognition between the two groups, and an additional nine antigens were recognized by a few patients in either of the two groups; but, again, as was the case for the adult antigen, no microfilarial antigen was recognized uniquely by the majority of ENs.

Infective larval (L3) stage antigen. In contrast to the findings with adult and microfilarial antigens, when reactivity of patient sera to antigens derived from infective larvae was examined (Fig. 4), the ENs were clearly more responsive than the MFs. The most striking difference between the two groups' responses was the recognition of a $43-\mathrm{kD}$ antigen (Fig. $4 \mathrm{~A}$, arrow) by $7 / 7(100 \%)$ of the ENs and only $1 / 12(8 \%)$ of the MFs $(P=0.0025)$ (Fig. $4 B)$. Even with overexposure of the autoradiographs, antigen strips incubated with the 11 nonreactive MF sera failed to show any recognition of the 43-kD antigen. The one MF who did recognize the 43-kD antigen had a microfilaremia of $2,513 \mathrm{mf} / \mathrm{ml}$ blood. No other major differences between the two groups were seen in the frequency-response analysis (Fig. 5).

That the 43-kD antigen was of filarial L3 origin and not an Aedes mosquito protein contaminating the L3 antigen preparation was demonstrated in the following two ways: first, when an immunoblot of crude Aedes aegypti extract antigen was probed with the $\mathrm{Wb}$ serum pool, no band at $43 \mathrm{kD}$ was recog- 
Table I. Study Population

\begin{tabular}{|c|c|c|c|c|c|c|c|c|c|}
\hline Patient & Sex & Age & Occupation & Stool O\&P & $\begin{array}{c}\mathrm{mf} \\
\text { Count }\end{array}$ & Total IgG & Antifilarial IgG & Total IgE & Antifilarial IgE \\
\hline & & $y r$ & & & No. $/ \mathrm{ml}$ & $m g / d l$ & $U / m l$ & $n g / m l$ & $n g / m l$ \\
\hline \multicolumn{10}{|c|}{ Endemic normals } \\
\hline 72 & $\mathrm{~F}$ & 65 & Domestic & + & 0 & 3,100 & 9,424 & 9,460 & 470 \\
\hline 76 & $\mathrm{~F}$ & 54 & Domestic & + & 0 & 2,420 & 6,048 & 8,800 & 95 \\
\hline 174 & $\mathbf{M}$ & 42 & Agriculture & + & 0 & 1,880 & 10,976 & 11,000 & 139 \\
\hline 176 & $\mathrm{~F}$ & 49 & Domestic & + & 0 & 1,640 & 7,920 & 1,760 & 91 \\
\hline 258 & $\mathbf{F}$ & 55 & Domestic & + & 0 & 2,232 & 9,736 & 7,000 & 540 \\
\hline 283 & $\mathbf{F}$ & 71 & Domestic & + & 0 & 1,680 & 22,912 & 4,400 & 53 \\
\hline 344 & $\mathrm{~F}$ & 55 & Domestic & + & $\underline{0}$ & $\underline{1,440}$ & $\underline{30,336}$ & 5,280 & $\underline{86}$ \\
\hline \multicolumn{4}{|c|}{ Geometric mean } & & 0 & 1,992 & 11,885 & 5,943 & 146 \\
\hline \multicolumn{10}{|c|}{ Microfilaremics } \\
\hline 5 & $\mathbf{F}$ & 71 & Domestic & + & 3,660 & 2,350 & 2,452 & 5,280 & 15 \\
\hline 47 & $\mathrm{~F}$ & 51 & Domestic & + & 2,513 & 2,100 & 4,414 & 2,420 & 11 \\
\hline 55 & $\mathrm{~F}$ & 45 & Domestic & + & 699 & 2,300 & 1,610 & 1,100 & 16 \\
\hline 92 & $\mathrm{~F}$ & 47 & Domestic & + & 1,710 & 1,900 & 730 & 2,640 & 5 \\
\hline 98 & $\mathbf{M}$ & 60 & Shopkeeper & + & 6,130 & 2,800 & 4,384 & 3,960 & 6 \\
\hline 112 & $\mathrm{~F}$ & 37 & Domestic & + & 81 & 2,200 & 6,464 & 8,360 & 28 \\
\hline 153 & $\mathbf{M}$ & 54 & Agriculture & + & 3,350 & 1,280 & 1,461 & 8,800 & 41 \\
\hline 209 & $\mathbf{M}$ & 39 & Agriculture & + & 75 & 1,960 & 2,720 & 1,320 & 14 \\
\hline 250 & $\mathbf{M}$ & 32 & Public works & + & 445 & 1,800 & 3,696 & 3,740 & 20 \\
\hline 348 & $\mathbf{F}$ & 58 & Domestic & + & 59 & 2,000 & 3,480 & 2,869 & 15 \\
\hline 366 & $\mathrm{~F}$ & 60 & Domestic & + & 586 & 1,600 & 2,704 & 2,000 & 26 \\
\hline 369 & $\mathbf{M}$ & 48 & Public works & + & 41 & $\underline{2,000}$ & $\underline{5,480}$ & $\underline{10,120}$ & $\underline{47}$ \\
\hline \multicolumn{5}{|c|}{ Geometric mean } & 556 & $\overline{1,900}$ & $\overline{2,838^{*}}$ & 3,474 & $\overline{17^{*}}$ \\
\hline
\end{tabular}

${ }^{*} P<0.001$

nized; second, when the $\mathrm{Wb}$ serum pool was absorbed multiply against Aedes antigens bound to nitrocellulose to the extent that it no longer could recognize essentially any Aedes

A

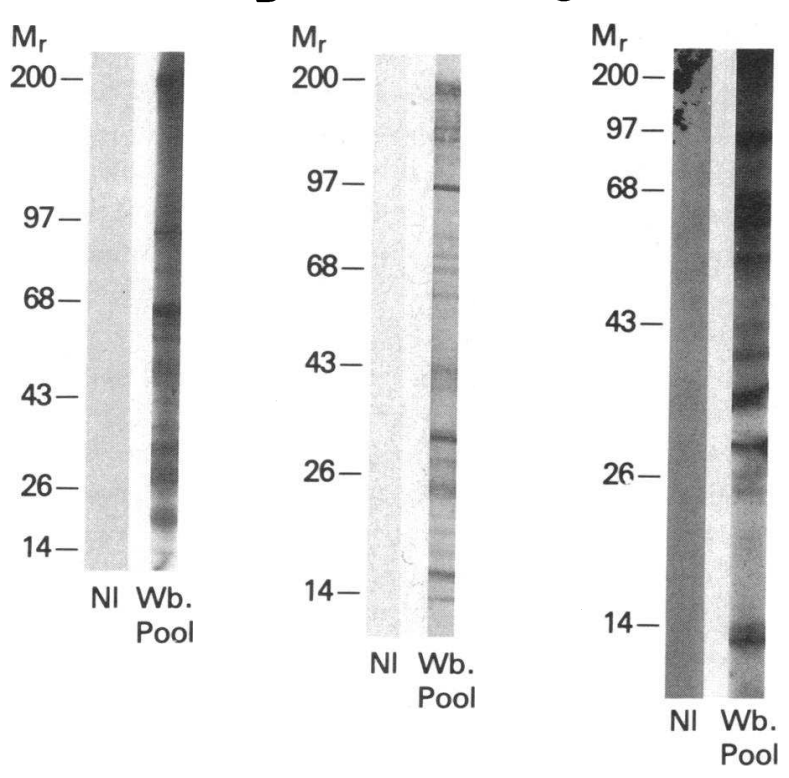

Figure 2. Immunoblot of IgG binding to $B$. malayi adult ( $A$ ), microfilarial $(B)$ and larval stage $(C)$ antigens by NHS (left strip in each panel) and the positive control Wb serum pool (right strip in each panel). antigens (Fig. 6, lane $B$ ), still it maintained its recognition of the $43-\mathrm{kD}$ band in the $\mathrm{L} 3$ antigen preparation (Fig. 6, lane $A$ ).

\section{Discussion}

There is an inherent ambiguity in attempting to define specific "protective antigens" for a human disease in which there exists no direct proof for the existence of protective immunity. The presence in areas hyperendemic for $W$. bancrofti or $B$. malayi of certain adults who are apparently infection-free (2) and the frequently observed leveling off of microfilaria rates in populations over the age of 30 (2) provide the best suggestive evidence available for naturally developing immunity. Despite extensive progress over the past several years on the characterization of filarial molecules immunogenic in both human and animal hosts (reviewed in 21), there remains to be answered the question of which of these molecules is relevant to the protection of humans from filarial infection.

In the present study we adopted an approach, similar to those taken with other parasitic organisms $(22,23)$, to identify protective filarial antigens; namely, a detailed comparative analysis of two separate groups of individuals at polar ends of the spectrum of protective immune responses to the parasite. At the protected end, a group of putatively immune infectionfree individuals (the ENs) was selected from a population with intense life-long exposure to bancroftian filariasis. In an attempt to find differences that might identify potentially protective antigens, immunoblot analysis was used to contrast the antigen recognition patterns of this putatively immune cohort with those of a group of definitely infected, presumably non- 


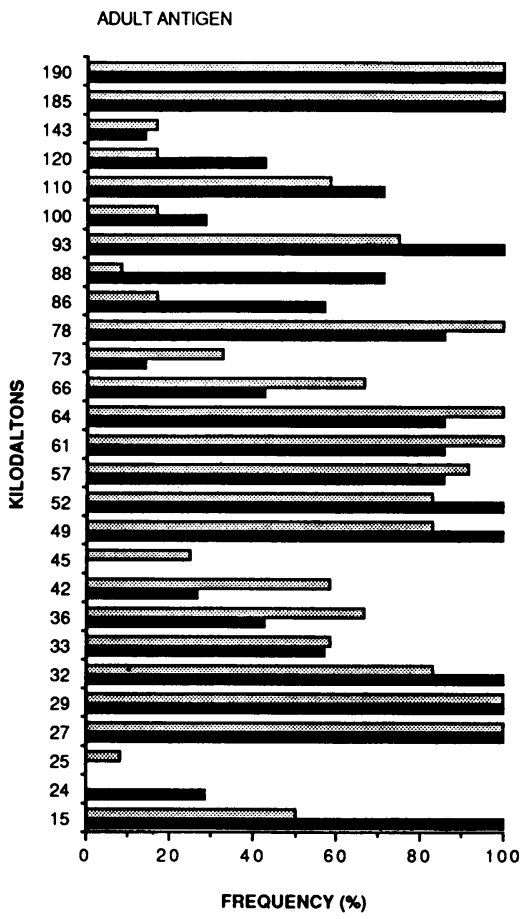

B

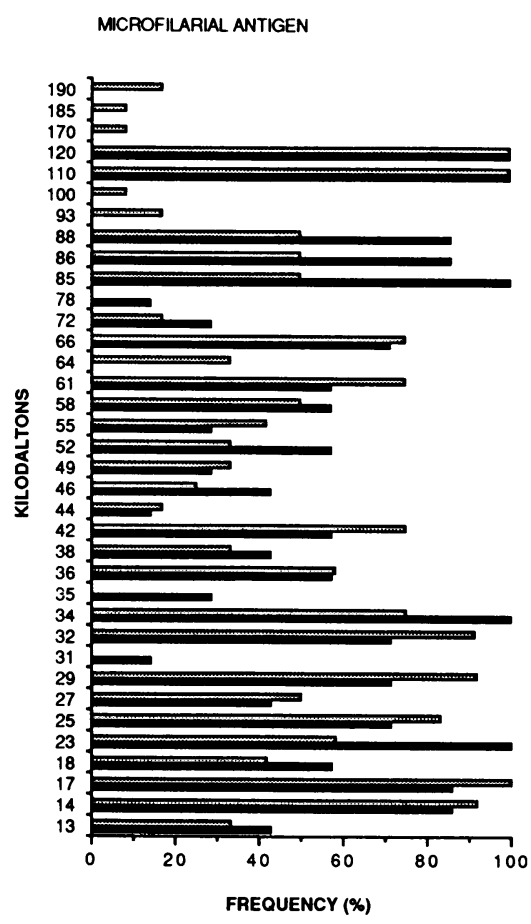

Figure 3. Frequency response profile of IgG antibody binding to $B$. malayi antigens of various molecular weights in ENs compared to MFs, as defined by immunoblotting. The $x$-axis shows the percentage of patients in each group that recognizes each antigen. $(A) B$. malayi adult antigen. (B) B. malayi microfilarial antigen. ENs (black bars); MFs (stippled bars). immune individuals (the MFs). That these MFs clustered at the presumably unprotected end of the spectrum is evidenced by their low filaria-specific humoral and cellular immune re- sponses, their high circulating antigen levels and by an absence of any clinical evidence of immune-mediated parasite destruction or host pathology.
A

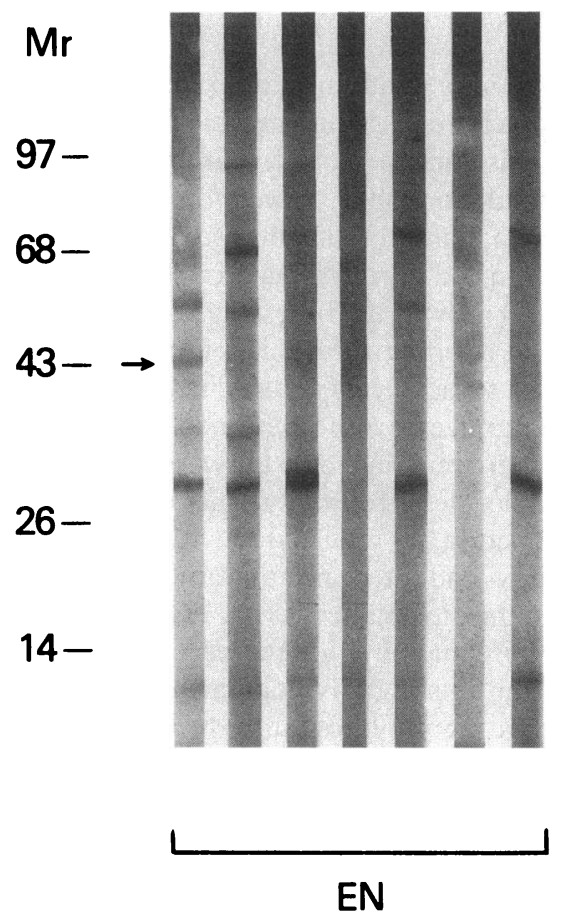

B

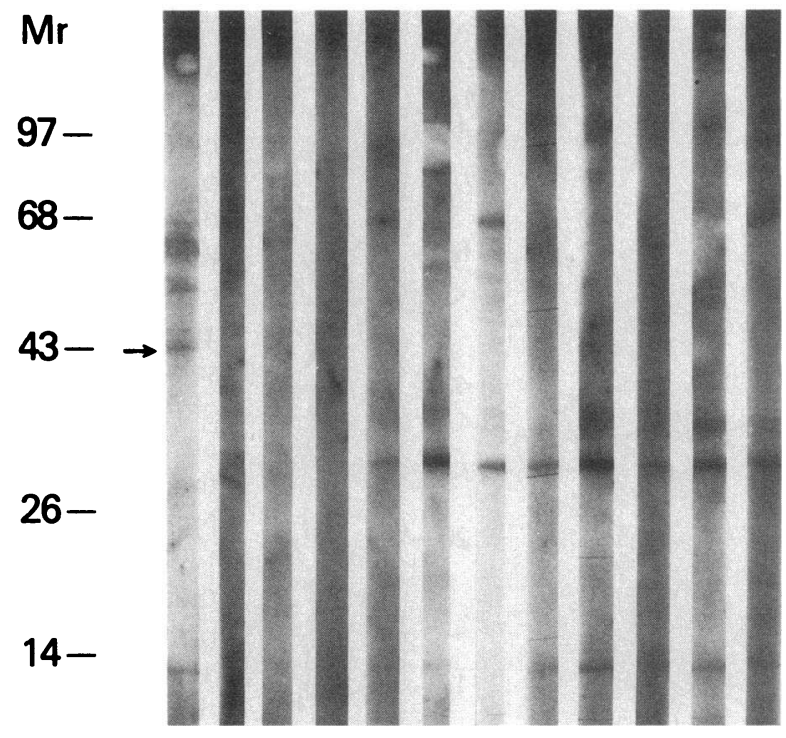

\section{MF}

Figure 4. Immunoblot of larval stage antigens (L3) recognized by patient sera. $(A)$ ENs. $(B)$ MFs. Arrow indicates $43-k D$ region. Positive and negative control sera lanes from this experiment are shown in Fig. $2 C$. 


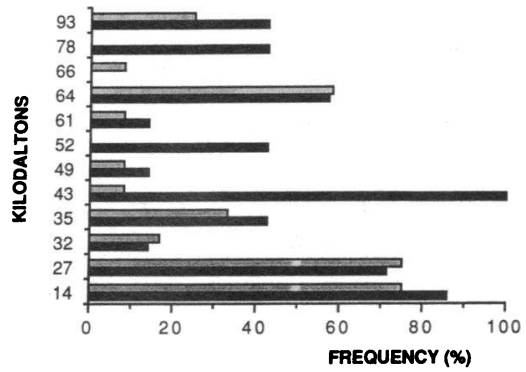

Figure 5. Frequency-response profile of $\operatorname{IgG}$ antibody binding to $B$. malayi larval stage antigens of various molecular weights in ENs and MFs. The $x$-axis shows the percentage of patients in the two groups that recognizes each antigen; ENs (black bars) MFs (stippled bars).

A major potential problem with this type of strategy, particularly for the human filarial infections is an inability to discriminate with certainty between amicrofilaremic individuals who are truly infection free and those who harbor occult infection (i.e., with living adult worms but no circulating microfilariae). The seven ENs selected as putatively immune for the present study each met the following strictest criteria we could formulate: $(a)$ an adult from a stable population intensively exposed to bancroftian filariasis; $(b)$ a negative history with a complete physical examination negative for stigmata of filarial disease; $(c)$ negative Nuclepore examinations for circulating microfilariae on at least seven occasions; $(d)$ no reaction to provocative or therapeutic doses of DEC; and, $(e)$ undetect-

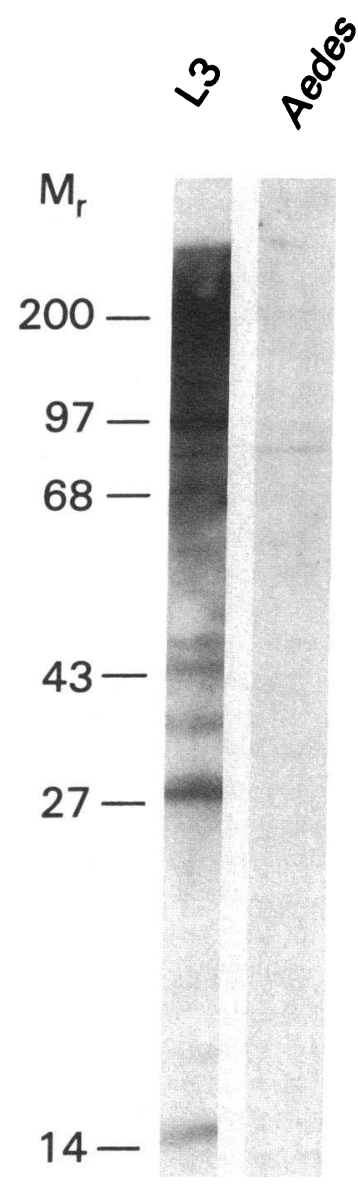

Figure 6. Immunoblot of the larval stage (L3) antigen preparation (lane $A)$ and an Aedes aegypti antigen preparation (lane $B$ ) with the $\mathrm{Wb}$. serum A B sool that had been previously ab- able levels of circulating filarial antigen in serum. Five other individuals met the first four criteria but were excluded from the study because of low level reactivity in the circulating antigen assay. Though persons with other helminth infections can sometimes have low levels of circulating PC antigen detectable in this assay (17) and despite the universal presence of intestinal helminths in our study population, it was felt that any detectable level of circulating antigen made that individual suspect for occult filarial infection and, thus, inappropriate for the study population. All of the $12 \mathrm{MFs}$, including the four with low-level microfilaremia ( $<100 \mathrm{mf} / \mathrm{ml}$ blood) had circulating antigen levels greater than $100 \mathrm{ng} / \mathrm{ml}$.

Qualitative analysis of IgG recognition patterns for each of the two patient groups, using the immunoblot technique with antigen prepared from the adult and microfilarial stages of the parasite, demonstrated complex banding patterns. Although there was inter-individual variation within each clinical group, the frequency-response analysis of Fig. 3 demonstrates clearly that the ENs recognized no unique adult or microfilarial stage antigens when compared with the MFs. In marked contrast, reaction of infective larval stage antigens with patient sera demonstrated the recognition of a unique 43-kD larval stage antigen by $100 \%(7 / 7)$ of the ENs compared to only $8 \%(1 / 12)$ of the MFs. Though it is still possible that some among the ENs actually harbored undetectable infection, the selective recognition of this $43-\mathrm{kD}$ antigen by those individuals who, by the most sensitive of criteria, appeared infection free despite intense exposure to the vector argues in favor of the existence of protective immunity with a potential role for this antigen in its induction. Four of the five individuals excluded from the EN group on the basis of elevated circulating antigen levels (Fig. 1) also recognized the $43-\mathrm{kD}$ antigen; thus, it may be that they did in fact belong in the EN group but were excluded because of low-level cross-reactivity in the circulating antigen assay induced by nonfilarial intestinal helminths (17).

To identify potentially significant differences in individuals' antigenic recognition patterns with the least ambiguity we examined only those persons felt to occupy positions at opposite ends of the protective immunity spectrum, despite the fact that animal models of lymphatic filariasis have clearly demonstrated that intermediate degrees of resistance may also exist and often be correlated with the presence of immunopathology. For example, repeated challenge infections given to cats infected with $B$. pahangi showed that partial resistance to reinfection (usually associated with the development of lymphatic immunopathology) could be seen even in animals remaining microfilaremic (6), and those animals converting to an amicrofilaremic state developed both greater lymphatic pathology and essentially complete resistance to reinfection (24). By analogy, one would anticipate that humans manifesting various degrees of immunopathology (i.e., clinical signs) caused by their filarial infection might also have different degrees of protective immunity (which would, of course, be impossible to quantitate in the absence of challenge studies); and if the $43-\mathrm{kD}$ antigen is causally related to protective immunity, heterogeneity in these individuals' responses to it would also be expected. When this possibility was examined in a group of individuals with immunopathology resulting from their filarial infections (i.e., episodic filarial fevers, hydrocele, lymphedema, or elephantiasis) heterogeneity in their antibody responses to the potentially protection-associated 43-kD larval stage antigen was, in fact seen. In contrast to ENs and MFs 
who reside at the clearly defined poles of the protective immunity spectrum, in this clinical group there was no consistent relationship between recognition of the $43-\mathrm{kD}$ antigen and the presence of circulating parasite antigen, the presence or absence of microfilaremia, or the levels of filaria-specific immunoglobulins (IgG or IgE). The fact that antibodies to the 43-kD antigen were seen in individuals both amicrofilaremic and microfilaremic (including one with $>300 \mathrm{mf} / \mathrm{ml}$ of blood) in this group with variable immunopathology argues against the possibility that the absence of such antibodies in our MF population merely reflects their passive absorption by circulating microfilariae. While seroreactivity to the $43-\mathrm{kD}$ antigen in a few individuals who clearly had both active infection (defined by microfilaremia) and evidence of immunopathology, might appear at odds with the hypothesis that recognition of this antigen is associated with the induction of protective immunity, the concept of concomitant immunity (whereby infected individuals are immune to reinfection), as has been demonstrated in schistosomiasis, would easily explain such findings. It is the inability to identify intermediate degrees of resistance to reinfection in these microfilaremic individuals with immunopathology that led us to study only those individuals with clearly definable degrees of protection (i.e., ENs and MFs).

It is not surprising, for at least two different reasons, that the putatively immune ENs recognize multiple antigens on the later (adult and microfilarial) life cycle stages of the heterologous parasite $B$. malayi used in our assays. First, more than 100 different polypeptides can be defined by two-dimensional gel electrophoresis on each of the three life cycle stages of $B$. malayi (25), the great majority of which are not stage specific; and observations on the limited numbers of available $W$. bancrofti adult parasites confirms the close antigenic similarity of these two filarial species (26). Second, the immunity putatively developed by these ENs (all of whom have $>40 \mathrm{yr}$ of exposure to the parasite) could just as reasonably have developed after patent infection had been immunologically cleared as it could have from de novo vigorous immune responses to infective larval antigens.

In view of animal and in vitro data suggesting a role for IgE in immunity against helminth infection (27-29) and in view of the high filaria-specific IgE levels in the EN group, IgE immunoblots were also performed (data not shown) to see whether the ENs mounted similar IgE responses to the potentially protective $43-\mathrm{kD}$ larval antigen. Though $0 / 12$ MFs produced detectable IgE against a 43-kD antigen, in only four of the seven ENs could this band be recognized. Therefore, evidence for a potential role for specific IgE in the differential recognition of this particular larval stage antigen in the EN group was equivocal.

As no concurrent entomologic studies were carried out in this population, the possibility that the putatively immune ENs had less intense exposure to infective L3s to explain their infection-free status must be entertained. Previously published epidemiological factors pertaining to Mauke argue against this possibility (12); parasite transmission occurred predominantly around the villages and there was no difference in filarial disease manifestations when the population was stratified according to indoor versus outdoor working conditions. In fact, filarial infection rates of day-biting Aedes polynesiensis, the principal vector of bancroftian filariasis in the Cook Islands, are greatest within and close to houses when compared to all other areas. Additionally, all four villages on this $2 \times 4$ mile island were similarly represented in both the EN and MF groups. Finally, the extremely elevated filaria-specific IgG and IgE levels in the ENs likely indicate persistent intense exposure to the parasite.

The reasons for the preponderance of females in the EN group (Table I) are uncertain. Prevalence rates of filarial infection stratified according to sex have not been systematically studied in any large population. It is known, however, that in some animal species (e.g., jirds [30]) females show preferential resistance to filarial infection. Differential susceptibility to filarial infection on a genetic basis has been investigated in only a few human studies. On Mauke there was clearly a familial clustering of disease but this was not linked to HLA-A or -B loci (HLA-DR was not studied), and predisposition to infection was most compatible with a genetically recessive inheritance pattern (31). Work with human filaria-specific $T$ cell clones has implicated a role for MHC genes in the induction of the antifilarial immune response (32); and in Sri Lanka and the Malay peninsula an association was reported between HLA B15 and chronic lymphatic disease in $W$. bancrofti infection (33). In mice, however, relative resistance to $B$. malayi microfilaremia has not been related to the $\mathrm{H}-2$ complex (34).

Because the injection of crude parasite material into human subjects is clearly not a feasible approach to the characterization of filarial molecules that have potential protective immunogenicity for humans, animal hosts that are permissive for human filarial parasites have been primarily utilized for vaccine studies. Evidence from several animal models in which the life cycles of Brugia species are reliably maintained indicates that a moderate degree of resistance to infection can be achieved by immunization with live, radiation-attenuated infective L3's $(35,36)$. However, the immunologic or biochemical nature of any protective antigens effecting immunity in these models has yet to be elucidated, a fact emphasizing the need for multiple alternative approaches.

Immune mediated clearance of the microfilarial stage of the parasite has been perhaps the most extensively characterized form of protection in animals immunized with nonliving parasite material. Jirds actively (37) or passively (38) immunized against the microfilarial stage of $B$. malayi rapidly clear microfilariae from the bloodstream, and distinct antigens can be implicated. This type of immunity does not seem to be efficient at preventing the development of adult worms after challenge infection with L3's in fully permissive hosts; however, in one study (37) the total adult worm burden at necropsy was significantly reduced in jirds vaccinated with a killed microfilaria extract. In addition, amicrofilaremic, but not microfilaremic humans from Papua New Guinea have been reported to recognize a $25-\mathrm{kD}$ microfilarial antigen of $B$. malayi that when used to immunize mice, induced clearance of microfilaremia (39). No similar differential recognition of an $\sim 25-\mathrm{kD}$ microfilarial antigen was found in our two (EN and MF) study groups.

Small animals permissive for human filarial parasites will remain a valuable tool for vaccine studies; if the effectors of the immunity induced by various immunological manipulations can be identified and the relevant protective epitopes isolated and synthesized, then human responses to them can be analyzed. Similarly, if potentially protective epitopes can be identified from studies in humans, their efficacy can only be assessed by studies in such animal models. It is in this context that our present study demonstrates the feasibility of a strategy 
to examine endemic populations to identify potentially protective antigens of relevance to human filariasis. Antisera are currently being raised to the $43-\mathrm{kD}$ larval stage antigen identified in this study after its isolation on two-dimensional gels. Utilization of these reagents should lead to the isolation, characterization, and molecular cloning of an epitope or epitopes that may be important in the induction of protective immunity to the lymphatic filarial infections of humans.

\section{Acknowledgments}

We would like to acknowledge the contributions of Drs. Peter Weller, Louis Heck, Tinika Tere and members of the staff of the Department of Health, Cook Islands whose participation was absolutely essential for the work on Mauke; to thank Dr. R. Lal for assistance with the circulating antigen assay; and to thank David Alling and Steven Banks for statistical advice.

\section{References}

1. World Health Organization Expert Committee on Filariasis. 1984. WHO (World Health Organization) Tech. Rep. Ser. No. 702.

2. World Health Organization. 1987. Protective immunity and vaccination in onchocerciasis and lymphatic filariasis: Report of the thirteenth meeting of the scientific working group on filariasis. WHO Document TDR/FIL/SWG(13)/87.3. Geneva.

3. James, S. L., and A. Sher. 1986. Prospects for a nonliving vaccine against schistosomiasis. Parasitology Today. 2:134-137.

4. Miller, L. H., R. J. Howard, R. Carter, M. F. Good, V. Nussenzweig, and R. S. Nussenzweig. 1981. Research toward malaria vaccines. Science (Wash. DC). 234:1349-1356.

5. Piessens, W. F., and C. D. Mackenzie. 1982. Immunology of lymphatic filariasis and onchocerciasis. In Immunology of Parasitic Infections. S. Cohen and K. S. Warren, editors. Blackwell Scientific, London. 622-652.

6. Denham, D. A., P. B. McGreevy, R. R. Suswillo, and R. Rogers. 1983. The resistance to re-infection of cats repeatedly inoculated with infective larvae of Brugia pahangi. Parasitology. 86:11-18.

7. Ottesen, E. A. 1984. Immunological aspects of lymphatic filariasis and onchocerciasis in man. Trans. R. Soc. Trop. Med. Hyg. 78(Suppl.):9-18.

8. Ottesen, E. A., P. F. Weller, M. N. Lunde, and R. Hussain. 1982. Endemic filariasis on a Pacific island. II. Immunologic aspects: immunoglobulin, complement and specific antifilarial IgG, IgM, and IgE antibodies. Am. J. Trop. Med. Hyg. 31:953-961.

9. Piessens, W. F., P. B. McGreevy, S. Ratiwayanto, M. McGreevy, P. W. Piessens, I. Koiman, J. Saroso, and D. T. Dennis. 1980. Immune responses in human infections with Brugia malayi: correlation of cellular and humoral reaction to microfilarial antigens with clinical status. Am. J. Trop. Med. Hyg. 29:563-570.

10. Ottesen, E. A., P. F. Weller, and L. Heck. 1977. Specific cellular immune unresponsiveness in human filariasis. Immunology. 33:413421.

11. Piessens, W. F., P. B. McGreevy, P. W. Piessens, M. McGreevy, I. Koiman, J. S. Saroso, and D. T. Dennis. 1980. Immune responses in human infections with Brugia malayi. Specific cellular unresponsiveness to filarial antigens. J. Clin. Invest. 65:172-179.

12. Weller, P. F., E. A. Ottesen, L. Heck, T. Tere, and F. A. Neva. 1982. Endemic filariasis on a Pacific island I. Clinical, epidemiologic, and parasitologic aspects. Am. J. Trop. Med. Hyg. 31:942-952.

13. McCall, J. W., J. B. Malone, L. R. Ash, and P. Thompson. 1973. Mongolian jirds (Meriones unguiculatus) infected with Brugia pahangi by the intraperitoneal route: a rich source of developing larvae, adult filariae and microfilariae. J. Parasitol. 59:436.
14. Rylatt, D. B., and C. R. Parish. 1982. Protein Determination on an automatic spectrophotometer. Anal. Biochem. 121:213-214.

15. Hamilton, R. G., R. Hussain, E. A. Ottesen, and N. F. Adkinson, Jr. 1981. The quantitation of parasite specific human IgG and IgE in sera: evaluation of a solid phase RIA and ELISA methodology. $J$. Immunol. Methods. 44:101-110.

16. Hussain, R., R. G. Hamilton, V. Kumaraswami, N. F. Adkinson, Jr., and E. A. Ottesen. 1981. IgE responses in human filariasis. I. Quantitation of filaria-specific IgE. J. Immunol. 127:1623-1629.

17. Lal, R. B., R. S. Paranjape, D. E. Briles, T. B. Nutman, and E. A. Ottesen. 1987. Circulating parasite antigen(s) in lymphatic filariasis: use of monoclonal antibodies to phosphocholine for immunodiagnosis. J. Immunol. 138:3454-3460.

18. Laemmli, U. K. 1970. Cleavage of structural proteins during the assembly of the head of bacteriophage T4. Nature (Lond.). 227:680-682.

19. Towbin, H.; T. Staehelin, and J. Gordon. 1979. Electrophoretic Transfer of proteins from polyacrylamide gels to nitrocellulose sheets: procedure and some applications. Proc. Natl. Acad. Sci. USA. 76:4350-4354.

20. Feller, W. 1968. An Introduction to Probability Theory and Its Applications. 3rd edition. John Wiley and Sons, New York. pp. 110.

21. Selkirk, M. E., D. A. Denham, F. Partono, I. Sutanto, and R. M. Maizels. 1986. Molecular characterization of antigens of lymphatic filarial parasites. Parasitology. 91:S15-S38.

22. Kemp, D. J., R. L. Coppel, A. F. Cowman, R. B. Saint, G. V. Brown, and R. F. Anders. 1983. Expression of Plasmodium falciparum blood-stage antigens in Escherichia coli: Detection with antibodies from immune humans. Proc. Natl. Acad. Sci. USA. 80:3787-91.

23. Dessein, A. J., M. Begley, C. Demeure, D. Caillol, J. Fueri, M. G. dosReis, Z. A. Andrade, A. Prata, and J. C. Bina. 1988. Human resistance to Schistosoma mansoni is associated with IgG reactivity to a 37kD larval surface antigen. J. Immunol. 140:2727-2736.

24. Denham, D. A., and P. B. McGreevy. 1977. Brugian filariasis: epidemiological and experimental studies. Adv. Parasitol. 15:243-309.

25. Lal, R. B., and E. A. Ottesen. 1988. Characterization of the stage-specific antigens of infective larvae of the filarial parasite Brugia malayi. J. Immunol. 140:2032-2038.

26. Morgan, T. M., I. Sutanto, Purnomo, Sukartono, F. Partono, and R. M. Maizels. 1986. Antigenic characterization of adult Wuchereria bancrofti filarial nematodes. Parasitology. 93:559-569.

27. Haque, A., W. Cuna, B. Bonnel, A. Capron, and M. Joseph. 1985. Platelet mediated killing of larvae from different filarial species in the presence of Dipetalonema viteae stimulated IgE antibodies. Parasite Immunol. 7:517-526.

28. Gusmao, R. A., A. M. Stanley, and E. A. Ottesen. 1981. Brugia pahangi: Immunologic evaluation of the differential susceptibility to filarial infection in inbred Lewis rats. Exp. Parasitol. 52:147-159.

29. Damonneville, M., C. Auriault, C. Verwaerde, A. Delanoye, R. Pierce, and A. Capron. 1986. Protection against experimental Schistosoma mansoni schistosomiasis achieved by immunization with schistosomula released products antigens (SRP-A): role of IgE antibodies. Clin. Exp. Immunol. 65:244-252.

30. Ash, L. R. 1971. Preferential susceptibility of male jirds (Meriones unguiculatus) to infection with Brugia pahangi. J. Parasitol. 57:777-780.

31. Ottesen, E. A., N. R. Mendell, J. M. MacQueen, P. F. Weller, D. B. Amos, and F. E. Ward. 1981. Familial predisposition to filarial infection-not linked to HLA-A or -B locus specificities. Acta Tropica. 38:205-216.

32. Nutman, T. B., E. A. Ottesen, A. S. Fauci, and D. J. Volkman. 1984. Parasite antigen specific $T$ cell lines and clones. Major histocompatibility complex restriction and B cell helper function. J. Clin. Invest. 73:1754-1762. 
33. Chan, S. H., S. Dissanayake, J. W. Mak, M. M. Ismail, G. B Wee, N. Srinivasan, B. H. Soo, and V. Zaman. 1984. HLA and filariasis in Sri Lankans and Indians. Southeast Asian J. Trop. Med. Publc. Health. 15:281-286.

34. Fanning, M. M., and J. W. Kazura. 1983. Genetic association of murine susceptibility to Brugia malayi microfilaremia. Parasite Immunol. 5:305-316.

35. Yates, J. A., and G. I. Higashi. 1985. Brugia malayi: vaccination of jirds with ${ }^{60}$ cobalt-attenuated infective stage larvae protects against homologous challenge. Am. J. Trop. Med. Hyg. 34:1132-1137.

36. Oothuman, P., D. A. Denham, P. B. McGreevy, G. S. Nelson, and R. Rogers. 1979. Successful vaccination of cats against Brugia pahangi with larvae attenuated by irradiation with $10 \mathrm{Krads}$ cobalt 60 . Parasite Immunol. 1:209-216.

37. Kazura, J. W.; H. Cicirello, and J. W. McCall. 1986. Induction of protection against Brugia malayi infection in jirds by microfilarial antigens. J. Immunol. 136:1422-1426.

38. Canlas, M., A. Wadee, L. Lamontagne, and W. F. Piessens. 1984. A monoclonal antibody to surface antigens on microfilariae of Brugia malayi reduces microfilaremia in infected jirds. Am. J. Trop. Med. Hyg. 33:420-424.

39. Kazura, J. W., H. Cicirello, and K. Forsyth: 1986. Differential recognition of a protective filarial antigen by antibodies from humans with bancroftian filariasis. J. Clin. Invest. 77:1985-1992. 